Cartagena, 7 a 9 de septiembre de 2011

\title{
Propuesta de un algoritmo genético para la programación diaria de los pedidos de una empresa del sector de la construcción
}

\section{A proposal of genetic algorithm for getting the daily scheduling of orders in a construction company}

\author{
Carola Pinto y Anna M. Coves \\ Instituto de Organización y Control de Sistemas Industriales y Departamento de Organización de Empresas \\ Universidad Politécnica de Catalunya. \\ anna.maria.coves@upc.edu
}

Fecha de recepción: 7-9-2011

Fecha de aceptación: 19-7-2011

\begin{abstract}
Resumen: El objetivo del presente trabajo es proponer un algoritmo genético para determinar la programación integrada de la producción y de la distribución de un producto de carácter perecedero y no almacenable, el hormigón. La empresa en cuestión cuenta con diferentes plantas productivas desde las cuales distribuye los pedidos hasta cada obra, con la ayuda de una flota de vehículos. La programación de los pedidos se efectúa el día anterior a su distribución, siendo el objetivo cumplir con el nivel de servicio acordado con el cliente y minimizar su coste asociado. El problema es NP-hard. Los resultados obtenidos muestran que el algoritmo genético propuesto realiza la programación minimizando costes y respetando las condiciones de entrega pactadas con el cliente.
\end{abstract}

Palabras clave: algoritmo genético, programación de la distribución del hormigón, programación de la producción del hormigón.

\begin{abstract}
The paper shows a genetic algorithm for making the production and delivery scheduling of a product non-storable and perishable, the ready mix concrete. The company in question has several factories from which delivery orders to each location, with the help of a fleet of trucks. The scheduling of orders is made the day before the delivery day, the aim being to meet the service level agreement with the customer and minimize the associated cost. This subject is a NP-hard problem. The algorithm genetic results of experimental study show that the algorithm obtains a schedule that minimize cost and taking into account the conditions agreed with the customer.
\end{abstract}

Key words: genetic algorithm, ready mix concrete distribution scheduling, ready mix concrete production scheduling.

\section{Introducción}

Los Algoritmos Genéticos (AGs) presentan una alternativa para solucionar problemas de optimización complejos. Si bien entenderlos es sumamente senciIlo, debido a que se asemejan a los procesos naturales que poseen las especies para su supervivencia, aplicarlos a un problema real implica la construcción de un sistema más complejo.

Se propone el diseño de un algoritmo genético para realizar la programación diaria de la producción y distribución de una empresa del sector de la construcción. El sector de la construcción necesita abastecimiento de materiales e insumos (cerámica, hor- migón, arena, etc.) que deben ser entregados, durante el día, dentro de plazos establecidos por cada cliente. El modelo parte de la base de un conjunto de cromosomas (soluciones iníciales) y utiliza cuatro operadores para hacer evolucionar a los cromosomas hacia una mejor solución. El objetivo es minimizar costes y cumplir con el nivel de servicio pactado con el cliente. Los costes involucrados corresponden al número de vehículos utilizados, a los costes operativos (tiempo, horas extras, etc.) y a los retrasos en las condiciones de entrega de los pedidos. Las condiciones de entrega consisten en pactar unos márgenes de entrega y una frecuencia de llegada de los vehículos que son necesarios para completar el volumen de un pedido. 
En los apartados siguientes se expone el escenario de la situación de partida, un esbozo del algoritmo desarrollado y las conclusiones del trabajo.

\section{Generalidades de los algoritmos genéticos}

Los algoritmos genéticos son algoritmos probabilísticos caracterizados por el hecho de que un número $\mathrm{N}$ de posibles soluciones (llamadas cromosomas), exploran el espacio de soluciones de un problema de optimización combinatoria (Michalewicz, 2004; Mitchell, 1996; Koza, 1990; Goldberg, 1988). Se desarrollaron tomando como base el proceso de evolución de los seres vivos y son parte de un grupo de algoritmos conocidos como algoritmos evolutivos que tienen como fortaleza llevar a cabo una búsqueda paralela en el espacio de soluciones.

Los AGs crean una población inicial de soluciones, cada una de las cuales tienen distinto grado de eficiencia. Una vez evaluada la eficiencia de cada solución se determina que soluciones serán seleccionadas para constituir la siguiente generación. Las poblaciones sucesivas se generan en base a distintos operadores. Una vez generada la siguiente población de cromosomas se mide la eficiencia de cada uno, si la mejor solución es adecuada, el proceso termina, caso contrario los operadores generan una nueva población y así hasta obtener una solución adecuada o se cumple la condición establecida de fin del algoritmo (tiempo de ejecución o no obtener mejora de la solución en un número determinado de generaciones, son las condiciones habituales).

A continuación se describen los cuatro operadores básicos utilizados:

- Selección: Por medio de este operador se selecciona a los mejores progenitores, probabilísticamente, para intentar generar mejores soluciones. Tras ser seleccionados estos progenitores se someten a las transformaciones producidas por el resto de operadores.

- Migración: La migración consiste en hacer que algunos cromosomas emigren de su población original y se trasladen a otra población.

- Cruzamiento: El cruzamiento simula a la reproducción humana. En las poblaciones este operador genera nuevos cromosomas. Usualmente se seleccionan dos cromosomas y estos producen nuevos descendientes, que representan nuevas soluciones.
- Mutación: La mutación consiste en pequeños cambios en las características de los cromosomas y tiene como objetivo introducir pequeñas transformaciones en la población que puedan derivar en mejores soluciones futuras.

El criterio utilizado para la finalización del algoritmo se determina en función de: un número de generaciones establecidas al inicio del algoritmo; la función de evaluación y un número determinado de generaciones en las que no se mejoran los resultados.

\section{Descripción del problema}

En el escenario aquí planteado una empresa programa la producción y distribución del día siguiente, que llamaremos día $D$. Se reciben los pedidos en el día D- I y al final de la jornada se programa la producción y distribución del día siguiente, día D, asignando los pedidos a las plantas. Así mismo se determina la flota de vehículos asignada a cada planta en función del número de pedidos y del volumen de servicio de la planta. También se realiza la programación de los viajes de los vehículos. A lo largo del día la demanda no es homogénea lo que origina una acumulación de vehículos en las colas de las plantas en determinas horas. (Coves et al., 2007; Naso et al., 2007). El problema abordado en este trabajo es NP-hard (Asbach et al., 2009) y (Yan Shangyao et. al., 2008)

\section{Plantas}

De cada planta se conoce la capacidad (en números de bocas de carga), su coste de producción, el tiempo de carga por vehículo, las jornadas laborales, horas y duración de los tiempos de descanso. Se conoce también la distancia entre cada planta-obra y la distancia entre todas las plantas.

\section{Pedidos}

Cada cliente registra su pedido, que corresponde a una obra, indicando el producto solicitado, el lugar de entrega, su volumen, la frecuencia de los envíos y se pacta la hora de inicio del servicio. En general se requieren varios viajes para entregar el volumen solicitado de un pedido. Se dispone de un margen, establecido previamente, para la hora de la primera entrega del pedido. Para las siguientes entregas del pedido se establece una determinada frecuencia. 


\section{Vehículos}

La empresa cuenta con una flota propia y cuando es necesario utiliza vehículos subcontratados. La remuneración a vehículos terceros se realiza en función al volumen distribuido o por horas trabajadas según el tipo de subcontratación. De cada vehículo se conoce su capacidad que varía entre 6 y $9 \mathrm{~m}^{3}$, siendo el de $6 \mathrm{~m}^{3}$ el más común en la empresa en cuestión en el periodo de realización del proyecto.

La carga de los vehículos en planta se rige a un sistema FIFO, de manera que a cada vehículo se le asigna un pedido cuando éste llega a la boca de carga.

Cada transportista tiene su periodo de descanso, en general entre $13 \mathrm{~h}$ y $\mid 4 \mathrm{~h}$, pero si en ese momento se encuentra en ruta esta hora de descanso se desplaza hasta el momento en que termine el servicio al cliente.

\section{Algoritmo genético AGPD-I}

Antes de iniciar el algoritmo genético para la programación de la producción y la distribución en el día D-I (AGPD-I), se deben consolidar los pedidos y generar el total de entregas del día siguiente (equivalente al número de viajes a realizar). Posteriormente se determina un número teórico de vehículos necesarios para atender esos pedidos, el algoritmo comienza su rutina partiendo de este número y una vez que la solución es encontrada se evalúa el impacto en costes.

\section{I. Los cromosomas}

Inicialmente, cada población es generada de manera aleatoria $(X=\{X \mid \ldots X n\})$. Cada solución candidata se codificará en un cromosoma. La representación de la soluciones es un problema no trivial y es clave en el desarrollo del proyecto. La idea fundamental es representar en una misma estructura todas las características del problema y asignar, mediante una función de evaluación, un solo valor que refleje la aptitud de cada instancia de dicha estructura.

Cada solución representada por un cromosoma deberá guardar la información necesaria para realizar la programación de la distribución. El cromosoma diseñado contiene tres partes diferenciadas: asignación de pedidos; asignación de prioridades y asignación de vehículos. Veremos un ejemplo para el caso de 3 plantas, 10 pedidos y 3 vehículos.

\section{- Primera parte:Asignación de Pedidos}

Cada gen es un número entero entre I y $D\{d$ $(I, \ldots D\}$, donde d representa el número de cada planta (Figura I), D = 3. Cada pedido es asignado a una planta y se representa con la letra r. Por ejemplo el pedido $\mid(r l)$ es asignado a la planta 2 (d2) (Figura I)

Figura I

Cromosoma Parte I

\begin{tabular}{|c|c|c|c|c|c|c|c|c|c|}
\hline$r 1$ & $r 2$ & $r 3$ & $r 4$ & $r 5$ & $r 6$ & $r 7$ & $r 8$ & $r 9$ & $r 10$ \\
\hline$d 2$ & $d 3$ & $d 2$ & $d 3$ & $d 2$ & $d 2$ & $d 3$ & $d 3$ & $d 1$ & $d 1$ \\
\hline
\end{tabular}

\section{- Segunda parte:Asignación de prioridades}

En el segundo bloque del mismo cromosoma, se considera la prioridad de entrega de los pedidos. Los valores van de $I$ a $P,\{P(I, \ldots P\}$, por ejemplo $P=10$, donde $p$ representa la prioridad del pedido, siendo el valor I el de máxima prioridad. En este caso el pedido r6 tiene máxima prioridad ( $\mathrm{pl}$ ) (Figura 2)

Figura 2

Cromosoma Parte II

\begin{tabular}{|l|l|l|l|l|l|l|l|l|l|}
\hline$p 1$ & $p 2$ & $p 3$ & $p 4$ & $p 5$ & $p 6$ & $p 7$ & $p 8$ & $p 9$ & $p 10$ \\
\hline$r 6$ & $r 2$ & $r 1$ & $r 8$ & $r 3$ & $r 5$ & $r 7$ & $r 10$ & $r 9$ & $r 4$ \\
\hline
\end{tabular}

\section{- Tercera parte:Asignación de vehículos}

En el tercer bloque del cromosoma se asignan los vehículos a las plantas, con valores entre $I$ y $\vee\{\vee(I, \ldots \vee\}$. Se ha considerado en el ejemplo que cada vehículo realiza un máximo de 5 viajes-día. Por ejemplo, v24 representa el cuarto viaje del vehículo 2, que es asignado a la planta d3 (Figura 3).

Figura 3

Cromosoma Parte III

\begin{tabular}{|c|c|c|c|c|c|c|c|c|c|c|c|c|c|c|}
\hline $\mathrm{vll}$ & $\mathrm{v} / 2$ & $\mathrm{v} / 3$ & $\mathrm{~V} \mid 4$ & 15 & V2I & V22 & 123 & v24 & v25 & V31 & V32 & v33 & & v35 \\
\hline d3 & $\mathrm{d} 2$ & d3 & d3 & d3 & d3 & $\mathrm{dl}$ & $\mathrm{d} 2$ & d3 & $\mathrm{dl}$ & d3 & $d l$ & $\mathrm{dl}$ & $d l$ & \\
\hline
\end{tabular}




\subsection{Evaluación de la función aptitud}

La determinación de la función de aptitud tampoco no es una tarea sencilla, como a priori no se conoce una función «buena» puede ser que existan problemas de múltiples óptimos locales y de aislamiento del óptimo global.

La función aptitud que se define, evalúa por una parte costes y por otra parte tiempos (retrasos y espera). Interesará minimizar el total de costes.

Costes: Se consideran el coste del transporte de la planta d a la obra del pedido r; el coste de viajes entre plantas.

Tiempos: Se considera el tiempo de demora en la entrega y los tiempos de espera de los vehículos en cola.

Para que la función pueda evaluarse se le asignó una penalización por cada minuto de demora en la entrega y por cada minuto que un vehículo espera para cumplir una nueva tarea. La obtención de esos costes asociados a los tiempos de demora y espera requiere de una simulación minuciosa de cada cromosoma para determinar en qué punto se generan demoras.

El proceso de simulación consiste en representar el circuito de actividades de cada cromosoma. Inicialmente se asignan los pedidos a las plantas, luego se asignan los viajes a los vehículos y finalmente se determinan los tiempos de demora y espera del circuito generado por el cromosoma. Todo este proceso se detalla en el apartado 4.3

\subsection{Simulación del cromosoma}

La simulación del cromosoma permite determinar para un cromosoma los valores de las variables que incluye la función aptitud, tales como: número de vehículos utilizados, programación de cada vehículo, horas extras de cada vehículo etc.

Paso I: Asignación de pedidos a plantas

Con los datos obtenidos en la primera parte del cromosoma se analiza el trabajo que cada solución le ha asignado a cada planta. Para todas las plantas se examinan las rutinas de trabajo que involucran los pedidos asignados. A su vez cada pedido contiene un número de viajes, con una hora de inicio en la entrega y frecuencias consecutivas.
Para cada viaje se determina la hora de inicio y de finalización de la tarea en función a los siguientes tiempos:

- Tiempo de carga en planta

- Tiempos de viaje a destino

- Tiempo de espera para la descarga

- Tiempo de descarga del producto

- Tiempo de retorno a la planta

Al finalizar el Paso I, se dispone de un listado de todas las tareas a realizar en cada una las plantas con horarios de inicio y finalización durante todo el día.

Paso 2: Asignación de viajes a los vehículos

La lista de tareas generada en el Paso I es ordenada de menor a mayor hora de inicio, con el objetivo de que los primeros viajes de cada vehículo sean realizados en las primeras horas del día. En la asignación de vehículos se considera la segunda parte del cromosoma, «Asignación de prioridades», pues puede haber pedidos que tengan el mismo horario de salida de una misma planta y no se disponga de suficientes bocas de carga en la planta.

Al finalizar el Paso 2, se dispone de un listado de todos los viajes que debe realizar cada vehículo.

Paso 3: Determinación de tiempos de demora y de espera

En función a la lista generada en el Paso 2 se simula el trabajo de cada vehículo y se determinan los tiempos totales en demora y espera para todos los vehículos involucrados.

Al finalizar el Paso 3 se dispone de la información necesaria para poder determinar el valor de la función aptitud del cromosoma.

\subsection{Los operadores}

\section{- Selección}

Mediante la selección determinamos los individuos de la población que tendrán descendencia. En el ejemplo expuesto en la (Figura 4) se tiene una población inicial de 10 individuos, los 10 han sido evaluados como posibles soluciones y presentan la función aptitud en la última columna. Los individuos se 
dividen en 2 grupos ( $G \mid$ y $G 2$ ), el $50 \%$ forma parte del grupo de los mejores individuos ( $\mathrm{Gl}$ ), es decir con la menor función aptitud y el restante $50 \%$ es el grupo (G2) con la función aptitud más elevada.
Del primer grupo se seleccionan aleatoriamente el $80 \%$, que para el ejemplo son 4 individuos y del segundo grupo se seleccionan el $40 \%$, es decir 2 individuos. Este grupo de 6 individuos es el nuevo gru-

Figura 4

Proceso de selección y creación de una nueva población

POBLACIÓN INICIAL

\begin{tabular}{|c|c|c|c|c|c|c|c|c|c|c|c|c|c|c|c|c|c|}
\hline$G$ & $P$ & $r l$ & r2 & r3 & r4 & r5 & $p l$ & p2 & p3 & $p^{4}$ & p5 & $\mathrm{vll}$ & $v 12$ & $v 13$ & $v \mid 4$ & $\mathrm{v} / 5$ & $\mathrm{FO}$ \\
\hline G2 & 1 & 2 & 3 & 2 & 3 & 2 & 6 & 2 & 1 & 8 & 3 & 1 & 2 & 2 & 2 & 3 & 675 \\
\hline G2 & 2 & 1 & 1 & 2 & 1 & 2 & 4 & 7 & 9 & 8 & 3 & 2 & 3 & I & 1 & 3 & 555 \\
\hline G2 & 3 & 3 & 2 & 3 & 3 & 2 & 3 & 5 & 8 & 2 & 9 & 3 & 3 & I & 2 & 2 & 750 \\
\hline GI & 4 & 2 & 3 & 1 & 3 & 2 & 8 & 10 & 5 & 1 & 4 & 2 & 2 & 2 & 1 & 1 & 170 \\
\hline GI & 5 & 2 & 3 & 2 & 2 & I & 4 & 7 & 5 & I & 10 & 3 & 3 & 3 & 3 & 1 & 305 \\
\hline GI & 6 & 1 & 1 & 3 & 1 & 1 & 5 & 8 & 4 & 7 & 3 & I & I & 2 & 2 & 2 & 275 \\
\hline G2 & 7 & 2 & 2 & 1 & 2 & 1 & 3 & 6 & 1 & 7 & 10 & 2 & 2 & 3 & 3 & 3 & 660 \\
\hline GI & 8 & 1 & 1 & 2 & 2 & 3 & 1 & 7 & 8 & 10 & 2 & 3 & I & 2 & 3 & 3 & 455 \\
\hline G2 & 9 & 3 & 2 & 2 & 1 & 1 & 9 & 3 & 2 & 10 & 6 & 1 & 1 & 3 & 2 & 3 & 610 \\
\hline GI & 10 & 1 & 1 & 3 & 1 & 3 & 7 & 4 & 6 & 5 & 1 & 2 & 3 & 3 & 2 & 2 & 495 \\
\hline
\end{tabular}

PADRES SELECCIONADOS

\begin{tabular}{|c|c|c|c|c|c|c|c|c|c|c|c|c|c|c|c|c|}
\hline $\mathrm{P}$ & $\mathrm{rl}$ & $\mathrm{r} 2$ & $\mathrm{r} 3$ & $\mathrm{r} 4$ & $\mathrm{r} 5$ & $\mathrm{pl}$ & $\mathrm{p} 2$ & $\mathrm{p} 3$ & $\mathrm{p} 4$ & $\mathrm{p} 5$ & $\mathrm{vlI}$ & $\mathrm{vl2}$ & $\mathrm{vl3}$ & $\mathrm{vl4}$ & $\mathrm{vl5}$ & $\mathrm{FO}$ \\
\hline 5 & 2 & 3 & 2 & 2 & $\mathrm{I}$ & 4 & 7 & 5 & $\mathrm{I}$ & 10 & 3 & 3 & 3 & 3 & $\mathrm{I}$ & 305 \\
\hline $\mathrm{I}$ & 2 & 3 & 2 & 3 & 2 & 6 & 2 & $\mathrm{I}$ & 8 & 3 & $\mathrm{I}$ & 2 & 2 & 2 & 3 & 675 \\
\hline 8 & $\mathrm{I}$ & $\mathrm{I}$ & 2 & 2 & 3 & $\mathrm{I}$ & 7 & 8 & 10 & 2 & 3 & $\mathrm{I}$ & 2 & 3 & 3 & 455 \\
\hline 10 & $\mathrm{I}$ & $\mathrm{I}$ & 3 & $\mathrm{I}$ & 3 & 7 & 4 & 6 & 5 & $\mathrm{I}$ & 2 & 3 & 3 & 2 & 2 & 495 \\
\hline 9 & 3 & 2 & 2 & $\mathrm{I}$ & $\mathrm{I}$ & 9 & 3 & 2 & 10 & 6 & $\mathrm{I}$ & $\mathrm{I}$ & 3 & 2 & 3 & 610 \\
\hline 4 & 2 & 3 & $\mathrm{I}$ & 3 & 2 & 8 & 10 & 5 & $\mathrm{I}$ & 4 & 2 & 2 & 2 & $\mathrm{I}$ & $\mathrm{I}$ & 170 \\
\hline
\end{tabular}

NUEVA POBLACIÓN

\begin{tabular}{|c|c|c|c|c|c|c|c|c|c|c|c|c|c|c|c|c|}
\hline$P$ & $r l$ & $r 2$ & r3 & r4 & r5 & $\mathrm{pl}$ & p2 & p3 & p4 & p5 & $\mathrm{vll}$ & $v 12$ & $v 13$ & $v \mid 4$ & vI5 & $\mathrm{FO}$ \\
\hline 1 & 2 & 3 & 1 & 3 & 2 & 8 & 10 & 5 & 1 & 4 & 2 & 2 & 2 & 1 & 1 & 170 \\
\hline 2 & 1 & 1 & 3 & 1 & 1 & 5 & 8 & 4 & 7 & 3 & 1 & 1 & 2 & 2 & 2 & 275 \\
\hline 3 & 2 & 3 & 2 & 2 & 1 & 4 & 7 & 5 & 1 & 10 & 3 & 3 & 3 & 3 & I & 305 \\
\hline 4 & I & 1 & 2 & 2 & 3 & 1 & 7 & 8 & 10 & 2 & 3 & 1 & 2 & 3 & 3 & 455 \\
\hline 5 & 3 & 2 & 2 & 2 & 1 & 4 & 7 & 5 & 6 & 2 & 2 & 3 & 3 & 3 & 1 & 345 \\
\hline 6 & 2 & 2 & 1 & 3 & 2 & 6 & 2 & 7 & 5 & 3 & 3 & 3 & 1 & 2 & 3 & 789 \\
\hline 7 & 1 & 3 & 2 & 2 & 3 & 10 & 6 & 8 & 10 & 2 & 3 & 1 & 2 & 2 & 3 & 567 \\
\hline 8 & 2 & 2 & 3 & I & 3 & 7 & 10 & 2 & 5 & 1 & 2 & 3 & 3 & 3 & 1 & 678 \\
\hline 9 & 2 & 3 & 2 & 1 & 1 & 1 & 4 & 2 & 10 & 6 & 1 & 1 & 2 & 2 & 2 & 302 \\
\hline 10 & 3 & 2 & 2 & 3 & 2 & 8 & 10 & 5 & 9 & 3 & 3 & 2 & 3 & 1 & I & 165 \\
\hline
\end{tabular}


po de progenitores sobre los que se ejecutan los operadores de cruce y mutación para generar nuevos individuos.

Finalmente la nueva población está compuesta por los 6 nuevos individuos que son los hijos de los progenitores originales y los 4 mejores individuos (40\%) de la población anterior. Esta nueva población es nuevamente evaluada para obtener la función aptitud de los nuevos individuos.

\section{- Cruzamiento}

Se tienen dos modalidades para este operador, en la primera se generan dos puntos de cruce aleatoriamente y se intercambian las subsecuencias centrales en el orden establecido (Figura 5).

El segundo procedimiento de cruce es empleado para la segunda parte del cromosoma (Figura 6). Ini- cialmente se generan 2 puntos de corte que dan lugar a dos subsecuencias (Reeves, 2010)

\subsection{Mutación}

La mutación es un operador que, a imitación de lo que ocurre en la naturaleza, fomenta la diversidad. La probabilidad de que una característica heredada (por ejemplo el valor de un gen) a un descendiente mute hacia otra, se establece a un nivel del 10\% (Figura 7).

La estructura general del algoritmo (Figura 8) sigue una línea secuencial donde en un principio se establecen los criterios y parámetros del algoritmo y posteriormente se utilizan todos los operadores para generar nuevas poblaciones.

Figura 5

Ejemplo de cruce para la primera y la tercera parte del cromosoma

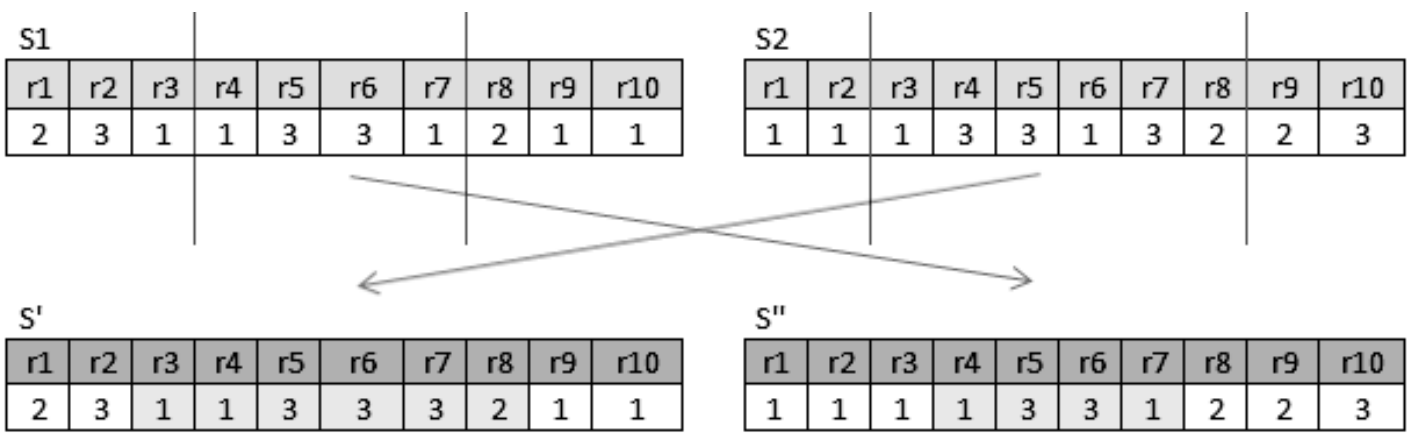

Figura 6

Modelo de Cruce para la segunda parte del cromosoma

SI

\begin{tabular}{|c|c|c|c|c|c|c|c|c|c|}
\hline$p 1$ & $p 2$ & $p 3$ & $p 4$ & $p 5$ & $p 6$ & $p 7$ & $p 8$ & $p 9$ & $p 10$ \\
\hline 2 & 4 & 5 & 6 & 9 & 7 & 1 & 8 & 3 & 10 \\
\hline
\end{tabular}

\begin{tabular}{|c|c|c|c|c|c|c|c|c|c|}
\hline$p 1$ & $p 2$ & $p 3$ & $p 4$ & $p 5$ & $p 6$ & $p 7$ & p8 & p9 & p10 \\
\hline 5 & 1 & - & - & 10 & 2 & - & 8 & 4 & 3 \\
\hline
\end{tabular}

S'

\begin{tabular}{|c|c|c|c|c|c|c|c|c|c|}
\hline$p 1$ & $p 2$ & $p 3$ & $p 4$ & $p 5$ & $p 6$ & $p 7$ & p8 & p9 & p10 \\
\hline 5 & 1 & 10 & 6 & 9 & 7 & 2 & 8 & 4 & 3 \\
\hline
\end{tabular}

S2

\begin{tabular}{|c|c|c|c|c|c|c|c|c|c|}
\hline$p 1$ & $p 2$ & $p 3$ & $p 4$ & $p 5$ & $p 6$ & $p 7$ & $p 8$ & $p 9$ & $p 10$ \\
\hline 5 & 1 & 7 & 9 & 10 & 2 & 6 & 8 & 4 & 3 \\
\hline
\end{tabular}

\begin{tabular}{|c|c|c|c|c|c|c|c|c|c|}
\hline$p 1$ & $p 2$ & $p 3$ & $p 4$ & $p 5$ & $p 6$ & $p 7$ & $p 8$ & $p 9$ & $p 10$ \\
\hline- & 4 & 5 & - & - & 7 & l & - & 3 & - \\
\hline
\end{tabular}

$S^{\prime \prime}$

\begin{tabular}{|c|c|c|c|c|c|c|c|c|c|}
\hline$p 1$ & $p 2$ & $p 3$ & $p 4$ & $p 5$ & $p 6$ & $p 7$ & $p 8$ & $p 9$ & $p 10$ \\
\hline 4 & 5 & 7 & 9 & 10 & 2 & 6 & 8 & l & 3 \\
\hline
\end{tabular}


Figura 7

Modelo de Mutación

\begin{tabular}{|c|c|c|c|c|c|c|c|c|c|}
\hline$r 1$ & $r 2$ & $r 3$ & $r 4$ & $r 5$ & $r 6$ & $r 7$ & $r 8$ & $r 9$ & $r 10$ \\
\hline 5 & 1 & 10 & 6 & 9 & 7 & 2 & 8 & 4 & 3 \\
\hline
\end{tabular}

\begin{tabular}{|c|c|c|c|c|c|c|c|c|c|}
\hline$r l$ & $r 2$ & $r 3$ & $r 4$ & $r 5$ & $r 6$ & $r 7$ & $r 8$ & $r 9$ & $r 10$ \\
\hline 5 & 1 & 10 & 6 & 7 & 9 & 2 & 8 & 4 & 3 \\
\hline
\end{tabular}

\subsection{Estructura del algoritmo genético AGPD-I}

Figura 8

Diseño del algoritmo genético AGPD- I

$$
i=0
$$

Generar lista de poblaciones iníciales (i)

Evaluar lista de poblaciones (i)

Hasta (condición de parada)

$$
\mathrm{i}=\mathrm{i}+\mathrm{I}
$$

Seleccionar m mejores poblaciones de la lista de población (i)

Realizar migraciones entre poblaciones

Re combinar en cada población de la lista de poblaciones (i)

Mutar en cada población de la lista de poblaciones (i) Evaluar cada población de la lista de poblaciones (i)

\section{Ejemplo}

\subsection{Datos, simulación del cromosoma y su función aptitud}

Para comenzar es necesario definir las condiciones iníciales del problema. Los elementos que conforman al problema y los parámetros necesarios para la ejecución del algoritmo. En la (Tabla I) se muestran los parámetros del ejemplo.

Tabla I

\section{Datos del ejemplo}

\begin{tabular}{|l|l|}
\hline Número de Pedidos & 10 \\
\hline Tamaño de la población & 100 \\
\hline Selección de padres & 60 padres seleccionados \\
\hline Migración & 40 mejores \\
\hline Tasa de mutación & $1 \%$ \\
\hline Criterio de parada & 30 repeticiones sucesivas \\
\hline
\end{tabular}

Los pedidos registrados en el día D-I son la base para la programación. El volumen de cada pedido y el número de viajes equivalente considerando un vehículo de 6 m3 de capacidad (Tabla 2). Se considera que se tienen 3 plantas productivas, cada una con 2 bocas de carga. El primer viaje puede tener un margen de +/- 15 minutos, sin embargo la frecuencia entre los viajes de un mismo pedido debe ser de $10 \mathrm{mi}$ nutos.

Tabla 2

\begin{tabular}{|c|c|c|c|c|}
\hline Cliente & Pedido & $\begin{array}{c}\text { Volumen } \\
\mathrm{m}^{3}\end{array}$ & Viajes & $\begin{array}{l}\text { Hora } \\
\text { en obra }\end{array}$ \\
\hline A & I & 11 & 2 & $9: 07$ \\
\hline B & 2 & 16 & 3 & $9: 06$ \\
\hline C & 3 & 8 & 2 & 10:06 \\
\hline D & 4 & 15 & 3 & $8: 14$ \\
\hline$E$ & 5 & 15 & 3 & $12: 18$ \\
\hline $\mathrm{F}$ & 6 & 16 & 3 & 14:05 \\
\hline G & 7 & 12 & 2 & $8: 08$ \\
\hline $\mathrm{H}$ & 8 & 6 & I & 15:07 \\
\hline I & 9 & 18 & 3 & $16: 11$ \\
\hline J & 10 & 2 & I & $9: 13$ \\
\hline & & & 23 & \\
\hline
\end{tabular}

Pedidos registrados en el día D-I

Se tiene un total de 23 viajes a realizar y se sabe que cada vehículo en promedio debe realizar 5 viajes al día, por lo tanto el número teórico de vehículos necesario es de 5 .

En base a estos parámetros se ejecuta el algoritmo genético AGPD- I y se obtiene el algoritmo con la mejor solución encontrada. 
Las tres partes del cromosoma presentadas en la Figura 9, 10 y II contienen la información necesaria para realizar el ciclo operativo.

Cada viaje de un pedido puede ser atendido desde cualquiera de las 3 plantas, pero no puede incumplir la frecuencia entre viajes definida en 10 minutos. La solución asigna los viajes y los pedidos a las plantas como se detalla (Tabla 3).

Tabla 3

Pedidos y viajes asignados

\begin{tabular}{|l|c|c|}
\hline \multicolumn{1}{|c|}{ Planta } & Pedidos & Viajes \\
\hline Planta I & 2 & 6 \\
\hline Planta 2 & 5 & 10 \\
\hline Planta 3 & 3 & 7 \\
\hline
\end{tabular}

Como restricción el número total de viajes asignados a cada planta debe ser mayor al número de viajes a realizar en esa misma planta. En caso de no cumplirse la condición se penaliza la solución con un número suficientemente alto de modo que disminuya la posibilidad de volver a ser elegido para la reproducción.

Los pedidos fueron asignados a las plantas como indica el Cromosoma Parte I: Asignación de pedidos. Por ejemplo, el pedido r4 y r5 fueron asignados a la planta I. En la (Tabla 4.I) se expone la asignación de los pedidos por planta con las respectivas horas y frecuencias de ejecución. La (Tabla 4.2) indica los tiempos de viaje entre planta y obra y la (Tabla 4.3) contiene, el cálculo del tiempo total para la primera entrega del pedido r4, como ejemplo ilustrativo.

Tabla 4.I

Tiempo de inicio y Fin de cada viaje

\begin{tabular}{|l|c|c|c|}
\hline \multicolumn{1}{|c|}{ Planta } & Pedido & Hora en PI & Hora de FV \\
\hline Planta I & 4 & $7: 50$ & $8: 34$ \\
\hline Planta I & 4 & $8: 00$ & $8: 25$ \\
\hline Planta I & 4 & $8: 10$ & $8: 44$ \\
\hline Planta I & 5 & $11: 50$ & $8: 35$ \\
\hline Planta I & 5 & $12: 00$ & $8: 54$ \\
\hline Planta I & 5 & $12: 10$ & $9: 21$ \\
\hline Planta 2 & 1 & $8: 50$ & $9: 28$ \\
\hline Planta 2 & 1 & $9: 00$ & $9: 32$ \\
\hline Planta 2 & 3 & $9: 50$ & $9: 31$ \\
\hline Planta 2 & 3 & $10: 00$ & $10: 00$ \\
\hline Planta 2 & 6 & $13: 50$ & $9: 48$ \\
\hline Planta 2 & 6 & $14: 00$ & $10: 22$ \\
\hline Planta 2 & 6 & $14: 10$ & $10: 32$ \\
\hline Planta 2 & 7 & $7: 50$ & $12: 25$ \\
\hline Planta 2 & 7 & $8: 00$ & $12: 35$ \\
\hline Planta 2 & 8 & $14: 50$ & $12: 45$ \\
\hline Planta 3 & 2 & $8: 50$ & $14: 21$ \\
\hline Planta 3 & 2 & $9: 00$ & $14: 31$ \\
\hline Planta 3 & 2 & $9: 10$ & $14: 41$ \\
\hline Planta 3 & 9 & $15: 50$ & $15: 24$ \\
\hline Planta 3 & 9 & $16: 00$ & $16: 29$ \\
\hline Planta 3 & 9 & $16: 10$ & $16: 39$ \\
\hline Planta 3 & 10 & $8: 50$ & $16: 49$ \\
\hline
\end{tabular}

Figura 9

\section{Cromosoma Parte I:Asignación de pedidos}

\begin{tabular}{|c|c|c|c|c|c|c|c|c|c|}
\hline$r l$ & $r 2$ & $r 3$ & $r 4$ & $r 5$ & $r 6$ & $r 7$ & $r 8$ & $r 9$ & $r 10$ \\
\hline 2 & 3 & 2 & 1 & 1 & 2 & 2 & 2 & 3 & 3 \\
\hline
\end{tabular}

Figura 10

\section{Cromosoma Parte II:Asignación de prioridades}

\begin{tabular}{|c|c|c|c|c|c|c|c|c|c|}
\hline$p 1$ & $p 2$ & $p 3$ & $p 4$ & $p 5$ & $p 6$ & $p 7$ & $p 8$ & $p 9$ & $p 10$ \\
\hline 7 & 9 & 3 & 2 & 6 & 4 & 1 & 10 & 8 & 5 \\
\hline
\end{tabular}

Figura I I

\section{Cromosoma Parte III:Asignación de vehículos}

\begin{tabular}{|c|c|c|c|c|c|c|c|c|c|c|c|c|c|c|c|c|c|c|c|c|c|c|c|c|}
\hline v1 & v12 & v13 & v14 & v15 & v21 & v22 & v23 & v24 & v25 & v31 & v32 & v33 & v34 & v35 & v41 & v42 & v43 & v44 & v45 & v51 & v52 & v53 & v54 & v55 \\
\hline 2 & 2 & 3 & 3 & 2 & 1 & 3 & 1 & 2 & 3 & I & 3 & । & 2 & 3 & 2 & 3 & 2 & 2 & 3 & I & 2 & 2 & I & 2 \\
\hline
\end{tabular}


Tabla 4.2

Tiempo de inicio y Fin de cada viaje

\begin{tabular}{|c|c|c|c|}
\hline Pedido & Planta I & Planta 2 & Planta 3 \\
\hline$r 1$ & 3 & 1 & 6 \\
\hline$r 2$ & 2 & 5 & 6 \\
\hline$r 3$ & 1 & 2 & 2 \\
\hline$r 4$ & 10 & 4 & 4 \\
\hline$r 5$ & 4 & 2 & 6 \\
\hline$r 6$ & 6 & 1 & 2 \\
\hline$r 7$ & 2 & 4 & 9 \\
\hline$r 8$ & 1 & 3 & 6 \\
\hline$r 9$ & 10 & 8 & 7 \\
\hline$r 10$ & 3 & 1 & 9 \\
\hline
\end{tabular}

Tabla 4.3

Resumen de tiempos p ejemplo del pedido 4

\begin{tabular}{|l|c|}
\hline Concepto & Tiempo \\
\hline Tiempo de carga & $14 \mathrm{~min}$ \\
\hline Tiempo de viaje planta-obra & $10 \mathrm{~min}$ \\
\hline Tiempo de espera descarga & $10 \mathrm{~min}$ \\
\hline Tiempo de descarga del material & $10 \mathrm{~min}$ \\
\hline Tiempo total & $44 \mathrm{~min}$ \\
\hline
\end{tabular}

El pedido $r 4$ expuesto en la (Tabla 4.1 ) registra un pedido de $15 \mathrm{~m}^{3}$, por lo tanto se subdivide en 3 viajes ( $15 \mathrm{~m}^{3}$ $16 \mathrm{~m}^{3}$ de capacidad $x$ vehículo). El primer viaje del pedido $r 4$ es realizado por un vehículo que comienza a cargar a horas 7:50, el vehículo carga durante 14 minutos en una de las dos bocas de carga de la planta I, luego a horas 8:04 comienza su viaje con destino a la obra, el vehículo viaja 10 minutos (Tabla 4.2) y a horas 8: 44 llega a la obra (hora pactada con el cliente), espera 10 minutos y a horas 8:24 descarga hasta las 8:34.

Una vez finalizado este primer viaje, el vehículo continúa con su itinerario (Tabla 5). Este viaje en total tuvo una duración de 44 minutos. (Tabla 4.3).

Una vez obtenidos los tiempos asociados a cada viaje se asigna los vehículos en función del Cromosoma Parte III: Asignación de vehículos. Todos los viajes se ordenan en función de la hora del pedido en obra. La regla de asignación es asignar los primeros viajes de todos los vehículos a los primeros pedidos que deben ser atendidos en el día.

El primer viaje del vehículo 2 «V2 I » es asignado a la planta I, el primer viaje del vehículo | «v | | a la planta 2 y así en función a la parte III del cromosoma. El vehículo v2 comienza su trabajo en la planta I, finaliza su primer viaje a horas 8:34 y se dirige a la planta 3 llegando a horas 8:38 (4 min de viaje), en la planta 3 espera 12 minutos para ser nuevamente cargado. De 8:50 a 9:28 realiza su segundo viaje con destino a la obra del pedido 2 y luego se dirige a la planta I Ilegando a horas 9:30 (2 min de viaje). El vehículo espera 2 horas con 30 minutos para ser cargado nuevamente. El tercer viaje se realiza entre las 12:00 y 12:35, luego a horas 12:37 el vehículo llega a planta 2, donde espera I hora con 13 minutos para ser cargado. Este procedimiento se repite con los restantes dos viajes de este y del resto de los vehículos.

Tabla 5

Asignación de vehículos

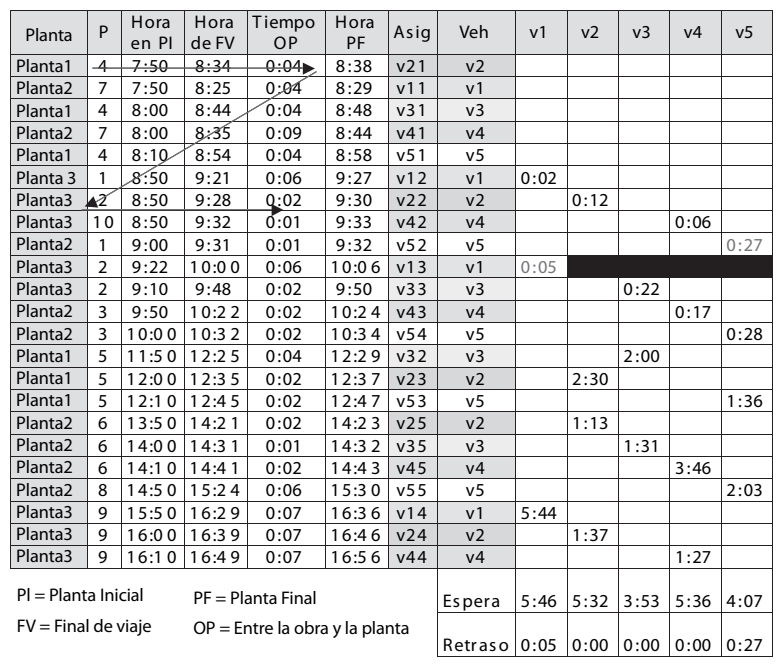

El tiempo total de espera de los vehículos en planta es de 1492 minutos y 32 minutos en demora en la entrega. Del mismo modo se analiza la carga de cada vehículo y en caso de superar las 9 horas de trabajo (incluye una hora de descanso) se calculan los tiempos extra de trabajo. En este caso ninguno de los vehículos supera las 9 horas.

\subsection{Coste asociado al tiempo de demora y espera}

A partir de la información obtenida en 5.I podemos determinar el coste correspondiente al cromosoma. La penalización por cada minuto de demora en la entrega es de 5 u.m./minuto y dado que se tienen 32 minutos de demora el resultado es un Coste por demora igual a 160 u.m.

Así mismo se penaliza la demora por la espera de vehículos en planta con 0,1 u.m./minuto. Como se 
tienen en total 1466 minutos de espera, el Coste por espera es 149,4 u.m.

Finalmente el minuto extra de trabajo es pagado con I,4 u.m., al no tener tiempos de trabajo extra es; Coste horas extra: 0 u.m.

\subsection{Coste asociado al transporte}

Los costes operativos de transporte asociados al viaje desde planta hasta la obra se determinan en función a la distancia y del coste unitario por kilometro recorrido. La (Tabla 6) muestra el resultado total de los costes referentes a este concepto.

Tabla 6

Costes de transporte Planta-Lugar de la obra

\begin{tabular}{|c|c|c|c|}
\hline Pedido & $\begin{array}{c}\text { Coste } \\
\times \text { viaje }\end{array}$ & $\begin{array}{c}\text { Número } \\
\text { de Viajes }\end{array}$ & $\begin{array}{c}\text { Coste } \\
\times \text { pedido }\end{array}$ \\
\hline 1 & 1,2 & 3 & 3,6 \\
\hline 2 & 7,2 & 3 & 21,6 \\
\hline 3 & 2,4 & 2 & 4,8 \\
\hline 4 & 12 & 3 & 36 \\
\hline 5 & 4,8 & 3 & 14,4 \\
\hline 6 & 1,2 & 3 & 3,6 \\
\hline 7 & 4,8 & 2 & 9,6 \\
\hline 8 & 3,6 & 1 & 3,6 \\
\hline 9 & 8,4 & 3 & 25,2 \\
\hline 10 & 10,8 & 1 & 10,8 \\
\hline & & & 133,2 \\
\cline { 4 - 5 } & & &
\end{tabular}

\subsection{Función aptitud}

La función aptitud está formada por cuatro tipos de coste (Tabla 7), un primer coste que tiene relación con la distancia recorrida, en este caso son 133, 4 u.m., un segundo coste corresponde a el tiempo de retrasos y espera 160 u.m. y I49,4 u.m. respectivamente. El último concepto de coste es el de coste por las horas extra de los transportistas, que para el ejemplo expuesto es 0 dado que todos los transportistas terminan su trabajo antes de las 8 horas de jornada laboral establecidas (Tabla 5).

La evaluación se realiza del mismo modo para cada una de las poblaciones de todas las iteraciones. Una vez obtenida la evaluación se continúa con los operadores de selección cruce y mutación siguiendo el ciclo de ejecución respectivo (Figura 12).
Tabla 7

Coste total de distribución según el cromosoma solución

\begin{tabular}{|c|c|}
\hline \multicolumn{2}{|l|}{ Coste de transporte } \\
\hline Coste de ir a la obra y volver a la planta & 133,4 \\
\hline \multicolumn{2}{|l|}{ Tiempos traducidos en costes } \\
\hline Coste por Retrasos & 160 \\
\hline Coste por Espera & 149,4 \\
\hline \multicolumn{2}{|l|}{ Coste de horas extra } \\
\hline \multirow[t]{2}{*}{ Horas extra } & 0 \\
\hline & 293,4 \\
\hline
\end{tabular}

\section{Resultados}

El algoritmo genético fue desarrollado en Visual Basic con interface en Microsoft Excel (versión 2007). El mínimo coste seleccionado como la mejor aptitud dentro de cada generación es representado en la (Figura 13). Se puede ver que la solución va evolucionando de manera decreciente consiguiendo cada vez un menor coste de operación y en la generación 48 encuentra la mejor solución que se repite 30 veces consecutivas hasta la generación 78, en este punto el algoritmo se detiene, según el criterio de parada, después de I hora con 16 min.

Finalmente la (Figura 14) expone la evolución de los padres en cada iteración y se comprueba una vez más que las operaciones utilizadas por el algoritmo generan mejores poblaciones.

\section{Conclusiones}

Mediante este estudio se ha podido corroborar la potencia de los algoritmos genéticos para resolver un problema de la programación de producción y distribución. Los resultados obtenidos muestran que se asignan pedidos y vehículos optimizando el uso de los recursos, disminuyendo el coste operativo y cumpliendo con el nivel de servicio pactado con el cliente, todo esto en un tiempo razonable.

Sin embargo el tiempo de procesado del algoritmo puede ser reducido, si en lugar de utilizar una interface gráfica como Excel durante todo el proceso, se la utiliza únicamente para visualizar resultados. El resto del proceso se haría en segundo plano.

Como líneas futuras, se propone añadir al modelos heurísticos que brinden una población de soluciones iníciales factibles, esto le permitiría al algoritmo genético comenzar la búsqueda a partir de un conjunto de buenas soluciones. 
Figura 12

Ciclo de ejecución del algoritmo AGPD-I

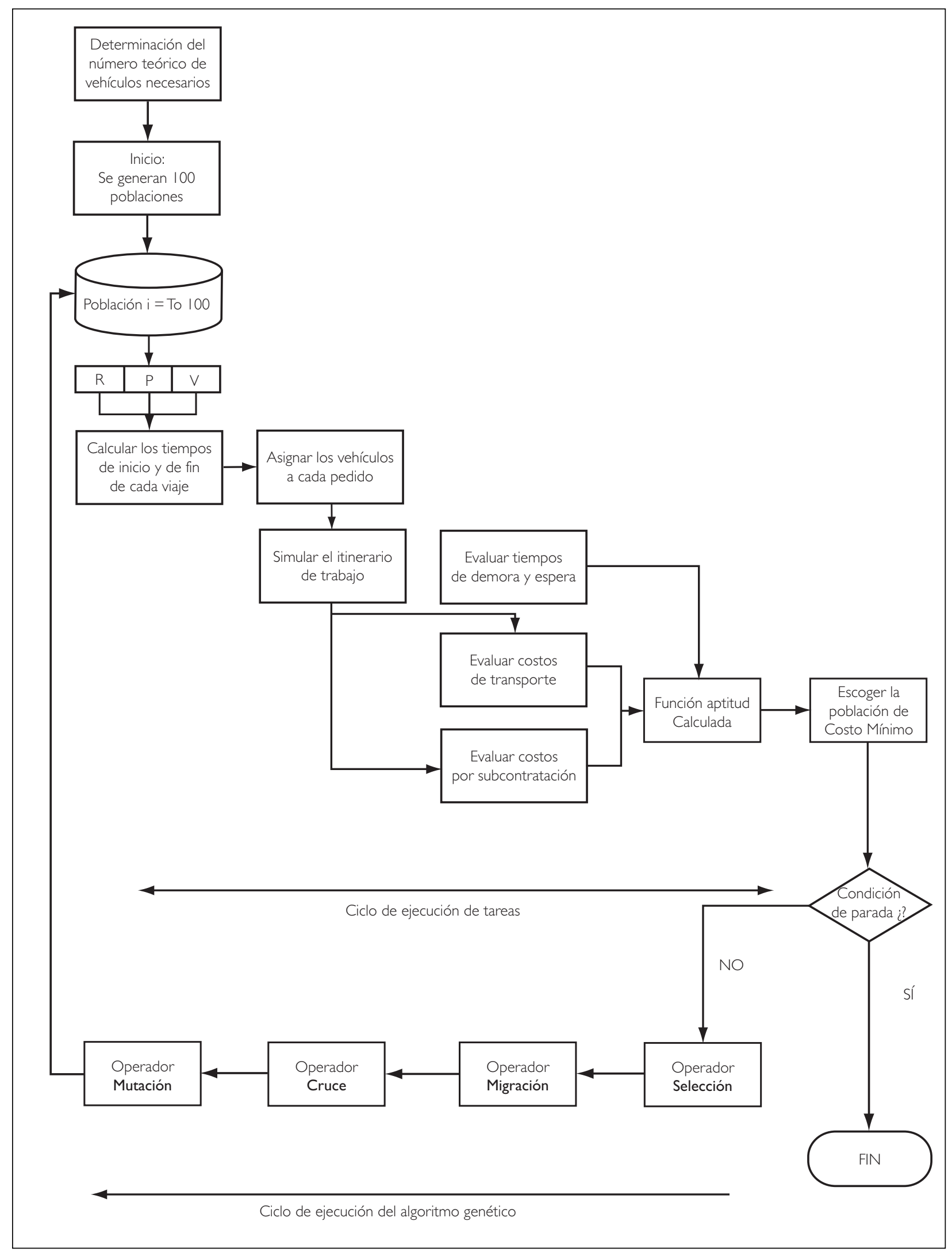


Figura 13

Evolución de la función aptitud

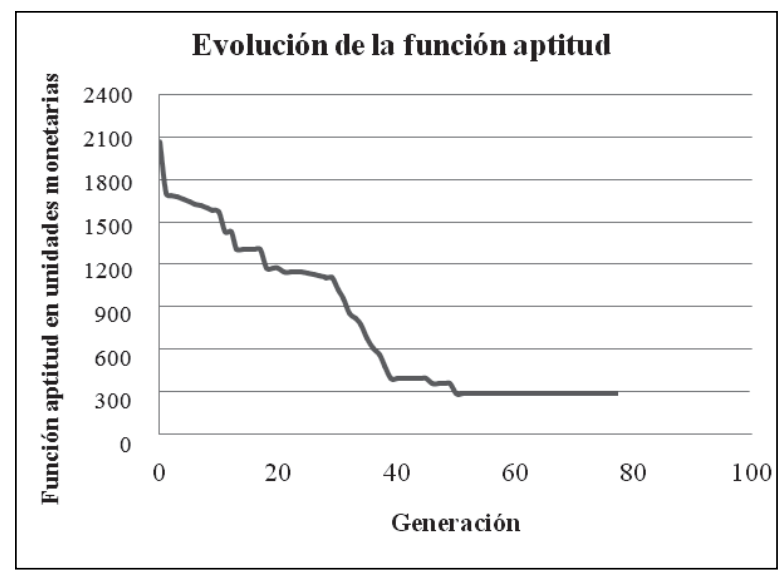

\section{Bibliografía}

ASBACH L, DORNDOF U., PESH E. (2009). Analysis, modeling and solution of the concrete delivery proble. European Journal of Operational Research, 193, pp. 820-835.

COVES A., DE LOS SANTOS M.A., MONTORI J, S PONS. (2007). Un caso de planificación integrada de la producción y la distribución de una empresa del sector de la construcción. Zaragoza. I er Congreso de logística y gestión de la cadena de suministro.

GOLDBERG, D. (1 988) Genetic Algorithms in Search, Optimization and Machine Learning, Addison-Wesley Pub.

KOZA, JR. (1990) Genetic Programming: A Paradigm for Genetically Breeding Populations of Computer Programs to Solve Problems. Stanford University Computer Science Department technical report.
Figura 14

Evolución media de los padres

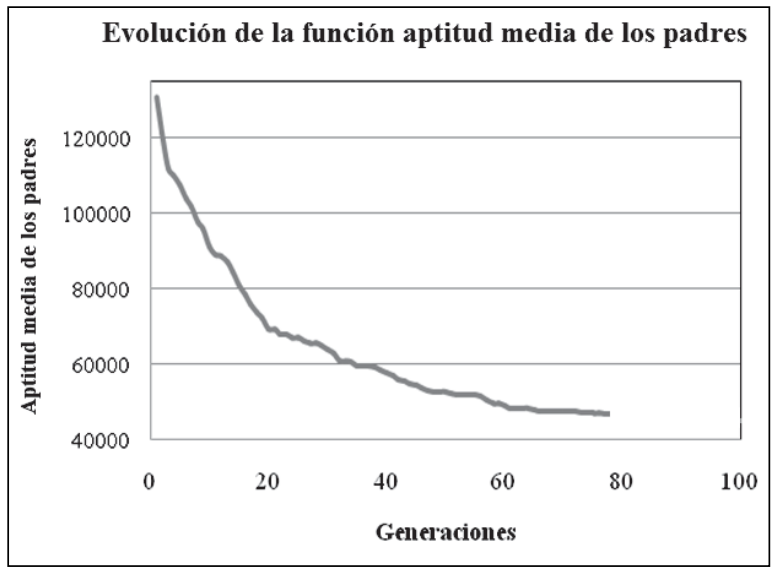

MICHALEWICZ Z.; FOGEL D.B. (2004) How to solve It: Modern Heuristics. Second edition. Springer.

MITCHELL, M. (1996) An Introduction to Genetic Algorithms. MIT Press.

NASO, D., SURICO, M., TURCHIANO, B., KAYMAK, U. (2007) Genetic algorithms for supply-chain scheduling: A case study in the distribution of ready-mixed concrete. European Journal of Operational Research, 177, pp. 2069-2099.

REEVES C.R. (2010) Hanbook of Metaheuristics. Second edition. Edited by Gendreau M.; Potvin J-Y. Springer. 5, pp. $109-140$.

YAN S., LAI W., CHEN M. (2006) Production scheduling and truck dispatching of ready mixed concrete. Transportation Research, Part E 44 (2008) 164- 179. 